Radiologe 2021 · 61:933-941

https://doi.org/10.1007/s00117-021-00898-0

Angenommen: 22. Juli 2021

Online publiziert: 7. September 2021

(๑) Der/die Autor(en) 2021

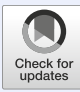

\title{
Strahlenanwendung in der Diagnostik von COVID-19
}

\section{Stellungnahme der Strahlenschutzkommission}

\author{
Strahlenschutzkommission (SSK) ${ }^{1,2}$ \\ 'Geschäftsstelle der SSK beim BfS, Bonn, Deutschland \\ ${ }^{2}$ Geschäftsstelle der Strahlenschutzkommission, Bonn, Deutschland
}

\section{Zusammenfassung}

Problem: Seit dem Auftreten der COVID-19-Pandemie wurde in einigen Veröffentlichungen vorgeschlagen, ionisierende Strahlung zur Diagnose einer COVID-19-Infektion einzusetzen. So soll die Computertomographie (CT) dafür eingesetzt werden, dass eine SARS-CoV-2-Infektion schon vor der symptomatischen Phase entdeckt wird.

Ziel der Beratung: Die Strahlenschutzkommission beschloss daher in ihrer 307. Sitzung am 2./3. Juli 2020 die Einsetzung einer SSK-Arbeitsgruppe, die sich mit den Strahlenschutzfragen im Zusammenhang mit einer COVID-19-Infektion befassen soll. Hierbei sollten insbesondere für den Strahlenschutz relevante Aspekte beim Einsatz einer Computertomographie zur Diagnostik einer COVID-19-Erkrankung erörtert werden. Der Beratung dieser Arbeitsgruppe, die ihre Arbeit am 5. Oktober 2020 aufnahm, liegt kein Beratungsauftrag des Bundesministeriums für Umwelt, Naturschutz und nukleare Sicherheit (BMU) zu Grunde, aber Vertreter des BMU und des Bundesamtes für Strahlenschutz begleiteten die Beratung unterstützend.

Stellungnahme: Die SSK nimmt wie folgt Stellung: 1. Die Anwendung einer CT bei asymptomatischen Personen zur Diagnose einer COVID-19-Erkrankung ist außerhalb von genehmigten Studien medizinisch nicht gerechtfertigt. 2. Gemäß 83 Abs. 3 Strahlenschutzgesetz erfordert auch eine CT zur Diagnostik und Verlaufskontrolle einer COVID-19-Pneumonie die vorherige Stellung der rechtfertigenden Indikation durch einen Arzt oder eine Ärztin mit der erforderlichen Fachkunde im Strahlenschutz. Diesbezüglich bieten die Empfehlungen der Fachgesellschaften aktuelle Hilfestellung.

\section{Schlüsselwörter}

Coronaviren · COVID-19·SARS-CoV-2 · Computertomographie $\cdot$ Strahlenschutz

\section{Einleitung}

Seit Anfang des Jahres 2020 hat die durch das Virus SARS-CoV-2 (engl.: "severe acute respiratory syndrome coronavirus type$2^{\prime \prime}$ ) verursachte Pandemie in Medizin und Gesellschaft tiefe Spuren hinterlassen. Einige der vielen Reaktionen auf die Bedrohung der durch das Virus verursachten Erkrankung COVID-19 (engl.: „coronavirus disease-2019") betreffen mittelbar oder unmittelbar Belange des Strahlenschutzes. So gibt es widersprüchliche Anregungen aus dem medizinisch-wissenschaftlich-technischen Bereich zum Einsatz von ionisierender Strahlung (primär der Computertomographie [CT]) bei der Diagnostik einer SARS-CoV-2-Infektion und der Verlaufskontrolle für eine COVID19-Erkrankung. Andere aktuelle Vorschläge beziehen sich auf eine Strahlentherapie der Lunge zur Behandlung von COVID19-Pneumonien mit schwerem Verlauf.

Aufgrund der Vielzahl der potenziell betroffenen Individuen und der damit verbundenen Größenordnung des Einsatzes ionisierender Strahlen in diesem Zusammenhang hält die Strahlenschutzkommission (SSK) eine Stellungnahme zu diesen beiden Anwendungsbereichen aus Sicht des Strahlenschutzes für erforderlich. Aus einer Sichtung der vorläufigen Evidenz sollen daher hier die Vor- und Nachteile der Anwendungsmöglichkeiten von Strah- 
lung in der Diagnostik und Therapie einer SARS-CoV-2-Infektion bzw. COVID-19Erkrankung herausgearbeitet und Empfehlungen zum sicheren Einsatz daraus abgeleitet werden. Mit Hinblick auf die Leserschaft dieser Zeitschrift beschränkt sich die vorliegende, gekürzte Fassung der Stellungnahme allein auf die diagnostische Strahlenanwendung, gibt sie aber im Übrigen unverändert wieder.

\section{Stellungnahme der SSK (gekürzt)}

Die SSK nimmt wie folgt Stellung:

- Die Anwendung einer CT bei asymptomatischen Personen ${ }^{1}$ zur Diagnose einer COVID-19-Erkrankung ist außerhalb von genehmigten Studien medizinisch nicht gerechtfertigt.

- Gemäß § 83 Abs. 3 Strahlenschutzgesetz [1] erfordert auch eine CT zur

\section{Abkürzungen}

ACE2-Rezeptor „Angiotensin-Converting

$A C R \quad$ American College of Radiolo-

COVID-19 "Corona Virus Disease-19"

CT Computertomographie

CTDIvol Volumen-CT-Dosisindikator

DRG Deutsche Röntgengesellschaft

KI Künstliche Intelligenz

LDCT Low-Dose-CT

LDRT "Low dose radiation therapy"

NHS National Health Service

NPV "Negative predictive value", negativer Vorhersagewert

PPV "Positive predictive value", positiver Vorhersagewert

$R C R \quad$ The Royal College of Radiologists

RNA "Ribonucleic acid", Ribonukleinsäure

RT-PCR "Reverse transcriptasepolymerase chain reaction", Reverse-TranskriptasePolymerase-Kettenreaktion

SARS-CoV-2 "Severe acute respiratory syndrome coronavirus type $2^{\prime \prime}$

WHO World Health Organization, Weltgesundheitsorganisation

\footnotetext{
1 In dieser Stellungnahme wird der Begriff „asymptomatisch“ füreineSARS-CoV-2-Infektion ohne Krankheitszeichen angewendet. Der Begriff ",asymptomatisch" wird hier nicht im Sinne der Begriffsbestimmung nach $\S 5$ Abs. 16 des StrlSchG verwendet, außer in Abschn. 4 "Rechtliche Rahmenbedingungen".
}

Diagnostik und Verlaufskontrolle einer COVID-19-Pneumonie die vorherige Stellung der rechtfertigenden Indikation durch einen Arzt oder eine Ärztin mit der erforderlichen Fachkunde im Strahlenschutz. Diesbezüglich bieten die Empfehlungen der Fachgesellschaften aktuelle Hilfestellung.

- Bei jeder Strahlenanwendung an COVID-19-Erkrankten sind zusätzlich zum Strahlenschutz ausreichende Maßnahmen zum Infektionsschutz des Personals zu ergreifen sowie Ressourcen für die medizinische Strahlenanwendung bezüglich anderer Indikationen, z. B. Diagnostik anderer schwerer Erkrankungen sowie Krebstherapien, zu gewährleisten.

\section{Wissenschaftliche Begründung (gekürzt)}

\subsection{Coronavirus-Pandemien}

Coronaviren stellen eine große Familie weit verbreiteter RNA-Viren dar, die vorwiegend die Epithelien des Magen-Darmund Atemwegstrakts infizieren können. Jede Coronavirus-Spezies zeigt eine Affinität zu einem bestimmten Säugetierwirt, beispielweise zu Hunden, Fledermäusen oder Schweinen. Die inhärente Mutabilität des viralen Genoms macht das Virus jedoch auch sehr anpassungsfähig an neue Wirtspezies. Die genetische Variabilität und die schnelle Entwicklung von mutierten Coronavirus-Stämmen sind für das Überleben des Virus vorteilhaft; dies spielt eine Rolle bei der bereits aktiven (intrinsischen) und der durch Infektion oder Impfung ausgelösten Immunabwehr des Wirts. Kürzlich wurden mutierte Varianten des SARS-CoV-2-Virus mit erhöhter Übertragungsrate bestätigt [2].

Der enge Kontakt des Menschen mit domestizierten und wilden Tieren, die Coronaviren tragen, hat zu einer Reihe von Infektionen mit Viren tierischen Ursprungs geführt. Coronaviren, die von tierischen Wirten auf Menschen übergegangen waren, haben in den letzten Jahren zu zwei Epidemien mit anhaltenden Infektionen in lokalisierten humanen Populationen geführt. Die erste Epidemie führte 2002 zu SARS (engl.: "severe acute respiratory syndrome") mit über 8000 Todesfällen in
26 Ländern [3]. Hierbei wird angenommen, dass die Infektion des Menschen über einen Fleckenmusang erfolgte, der mit einem Coronavirus der Fledermaus infiziert war. Die zweite Coronavirus-Epidemie, die zu MERS (engl.: "Middle East respiratory syndrome") führte, trat 2004 auf, wobei Kamele als wahrscheinlicher Überträger zwischen Fledermäusen und Menschen identifiziert wurden. In diesem Fall blieb die Infektion durch ihre geographische Lage auf die arabische Halbinsel beschränkt, und es wurden weniger als 3000 Todesfälle gemeldet [4]. Obwohl SARS verschwunden zu sein scheint, ist das MERS-Virus gelegentlich in der Golfregion wiederaufgetaucht. Es wurde vorhergesagt, dass die zunehmende Globalisierung weitere Krankheitsausbrüche durch Infektionen mit Coronaviren begünstigen wird, was sich nunmehr mit dem COVID-19-Ausbruch bestätigt hat [5].

Obwohl der Zwischenwirt der aktuellen COVID-19-Pandemie bisher nicht sicher ausgemacht werden konnte, ist ein erneuter Ursprung bei Fledermäusen wahrscheinlich. SARS-CoV-2 hat sich bei der Besiedlung der Atemwege als äußerst effizient erwiesen und ist mit einer signifikanten Morbidität verbunden. Die aktuelle pandemische Erkrankung COVID-19 manifestiert sich häufig mit einer Entzündung der oberen Atemwege mit trockenem Husten und Fieber sowie anderen, z. B. neurologischen Symptomen (Verlust des Geschmacks- und Geruchssinns). Neben leichteren Verläufen kommen auch schwere Verläufe mit interstitieller Pneumonie vor. Die Ansteckungsgefahr beginnt bereits etwa zwei Tage vor Symptombeginn. Ein Teil der Patienten und Patientinnen erkrankt schwer und wird intensiv- und/oder beatmungspflichtig. In schwereren Fällen verläuft die Erkrankung typischerweise in mehreren Phasen, wobei sich die Symptomatik des oberen Atemtrakts vorübergehend bessern kann und um den siebten bis zehnten Krankheitstag Symptome der Pneumonie auftreten können, mit dem Leitsymptom der Atemnot. Darüber hinaus hat sich gezeigt, dass COVID-19 nicht nur eine Atemwegs- und Lungenerkrankung ist, sondern u.a. auch das zentrale Nerven- und kardiovaskuläre System, den Gastrointestinaltrakt und die Blutgefäße betreffen kann. Insbesondere schwere, tödliche Verläufe zeigen oft 
Lymphopenie, Thrombosen in terminalen Gefäßen, Myokarditis und eine inadäquate Immunreaktion mit einem Zytokinsturm und einer überschießenden Entzündungsreaktion in einer Vielzahl von Organen [6-8].

Die Epidemie wurde am 11. März 2020 von der Weltgesundheitsorganisation (WHO) als Pandemie eingestuft. Die hohe Infektionsrate hat die Gesundheitssysteme in vielen Ländern überfordert. Die WHO meldete im Rahmen der COVID19-Pandemie bis Anfang Februar 2021 weltweit kumulativ 106 Mio. Fälle und 2,3 Mio. Todesfälle. Aktuelle Zahlen sowie der Verlauf der Pandemie sind auf der Internetseite der WHO unter "https:// covid19.who.int" [9] zu finden.

In Deutschland wurden bis Anfang Februar 2021 ca. 2,3 Mio. Fälle und ca. 62.000 Todesfälle registriert. Auch hierzu finden sich die aktuellen Zahlen und der Verlauf der Pandemie im Internet auf der Seite:

https://www.rki.de/DE/Content/InfAZ/ N/Neuartiges_Coronavirus/Fallzahlen.html [10].

Obwohl erwartet wird, dass Schutzmaßnahmen und Impfkampagnen die Auswirkung in den kommenden Monaten verringern werden, muss berücksichtigt werden, dass sich in Zukunft möglicherweise therapie- bzw. immunitätsresistente SARS-CoV-2-Varianten entwickeln und dass langfristig weitere Epidemien mit anderen Coronaviren ernsthafte Gesundheitsrisiken darstellen werden.

\subsection{Anwendung strahlenbasierter bildgebender Verfahren bei COVID- 19-Infektionen}

\subsubsection{Aktuelle Diagnostik}

Derzeitiger Standard der Diagnose COVID19 ist die Reverse-Transkriptase-Polymerase-Kettenreaktion (engl.: "reverse transcription polymerase chain reaction", RT$P(R)$ aus einem Rachenabstrich, der transoral oder transnasal gewonnen wird, um Virus-RNA nachzuweisen. Die Sensitivität dieser Methode hängt u. a. von der Qualität des Abstrichs ab, aber auch von der Phase des Infektionsverlaufs. Wenn die Entzündung der oberen Atemwege abgeklungen ist, kann die RT-PCR aus dem Rachenabstrich falsch-negativ ausfallen, obwohl die Infektion sich zur gleichen Zeit bereits im unteren Atemtrakt manifestiert hat $[6,7]$. Sputum, aus dem sich Virus-RNA isolieren ließe, wird von einigen Patienten und Patientinnen nicht produziert. Bei diesen wäre für den Nachweis mittels RT-PCR die Gewinnung von Bronchialsekret erforderlich, d.h. über eine flexible Bronchoskopie, eine bronchoalveoläre Lavage oder eine Absaugung bei intubierten Personen - alles Maßnahmen, die nicht nur invasiv sind, sondern auch mit einem erheblichen $\mathrm{Ri}$ siko für eine Infektion des medizinischen Personals behaftet sind.

Logistik und laborinterne Abläufe führen dazu, dass die Ergebnisse von RT-PCRTests gewöhnlich erst am nächsten Arbeitstag verfügbar sind, oft auch erst nach mehreren Tagen. Dies stellt einen schwerwiegenden Nachteil der Methode dar. Zudem wird für den Fall einer stark steigenden Zunahme der Krankheitsinzidenz befürchtet, dass Labormaterialien weltweit knapp werden könnten - dies betrifft weniger Reagenzien als allgemeiner Laborbedarf wie Pipettiermaterialien, Plastikröhrchen etc. In der Praxis verzögert dies Diagnostik- und Behandlungsabläufe, verlängert die Zeit, in der Dritte angesteckt werden können, und blockiert Kapazitäten im Gesundheitssystem.

Seit dem Herbst 2020 sind SARS-CoV-2Antigen-Schnelltests breit verfügbar. Mit derartigen Tests können Interessierte an Ort und Stelle getestet werden und ein Ergebnis liegt innerhalb von $30 \mathrm{~min}$ vor [11]. Die diagnostische Genauigkeit genügt jedoch nicht allen Ansprüchen. Die Aussagekraft hängt davon $a b$, ob eine Erkrankung besteht und welche Antigenkonzentration vorliegt, und nicht zuletzt ist sie abhängig von der Qualität und dem Zeitpunkt der Probengewinnung. Die Sensitivität wird mit ca. $90 \%$ und die Spezifität wird mit ca. $95 \%$ angegeben, allerdings handelt es sich um Angaben der Hersteller zur Güte der Tests im Labor, nicht beim Einsatz in der Praxis. Die Forschergruppe „Diagnostics Global Health" um das Institut für Tropenmedizin der Universität Heidelberg hat die aktuell verfügbaren, unabhängigen Studien - teils bereits begutachtet, teils noch im "Pre-Print"-Stadium - zur Sensitivität und Spezifität kommerzieller Schnelltests systematisch überprüft und auf das Risiko einer systematischen Verzerrung (Bias) hin bewertet. Eine Übersicht über die Ergebnisse einschließlich Links zu den Originalpublikationen findet sich unter: https://diagnosticsglobalhealth.org [12]. Darin bestätigt sich weitgehend die von den Herstellern angeführte hohe Spezifität, während die Sensitivität der Tests breit streut und teilweise deutlich niedriger ausfällt. So liegt die Mehrzahl der Angaben zwischen 70 und $85 \%$. Hierbei ist zu berücksichtigen, dass Schnelltests nicht geeignet sind nachzuweisen, dass eine Person infiziert ist, sondern nur, ob die getestete Person gerade Viren produziert und damit infektiös ist. Falls bereits eine Infektion vorliegt, können die Getesteten auch bei negativem Schnelltest sehr schnell infektiös werden. Schnelltests sind für die Testung asymptomatischer Personen gedacht und können $z$. B. verwendet werden, um den Zutritt einer positiv getesteten Person zu einem gefährdeten Bereich (z. B. einem Pflegeheim) zu verhindern und eine RT-PCR zu veranlassen.

\subsubsection{Rationale 2 für den Einsatz der Computertomographie der Lunge bei COVID-19-Verdacht}

Während die Projektionsradiographie gegenüber pulmonalen Veränderungen wenig sensitiv ist, zeigt die native Computertomographie inklusive der "Low-dose"-CT (LDCT) typische Befunde einer beidseitigen, überwiegend multifokalen interstitiellen Pneumonie bei COVID-19-Erkrankten [13], die umso stärker ausgeprägt sind, je schwerer die Patienten und Patientinnen klinisch erkrankt sind [14, 15]. Am häufigsten finden sich:

- Milchglasverdichtungen,

- Konsolidierungen,

- „inverse halo sign“: Milchglasverdichtung, umgeben von einer ringförmigen Konsolidierung,

- verdickte Interlobulärsepten und

- "crazy paving": eine Kombination aus Milchglasverdichtung und verdickten Septen.

Durch standardisierte Befundungskriterien kann die diagnostische Aussagekraft derCT erhöht werden [16]. Gleichwohl sind diese Befunde für die COVID-19-Pneumo-

\footnotetext{
2 Rationale: wird in dieser Stellungnahme als begründende Überlegung verstanden
} 
nie keineswegs spezifisch, sondern werden auch z. B. bei viralen Pneumonien anderer Ursache nachgewiesen, so z.B. bei der Grippepneumonie oder der eosinophilen Pneumonie. Manche Befunde sind für eine COVID-19-Pneumonie weniger typisch, so z. B. „Tree-in-Bud"-Phänomene (feines, dendritisches Muster, das eine Erkrankung der terminalen Atemwege anzeigt). Kavitationen oder Pleuraergüsse können aber in späteren Phasen der Erkrankung und insbesondere bei bakterieller Superinfektion auftreten. Verschlüsse pulmonalarterieller Äste infolge spontaner Thrombosen oder Embolien aus einer Quelle im großen venösen Kreislauf können nur mit der konstrastverstärkten CT nachgewiesen werden.

Berichten aus der frühen Phase der Pandemie zufolge sind pathologische Befunde in der Nativ-CT des Thorax häufig, und zwar auch bei Patienten und Patientinnen mit nur leichter bis mäßiger Symptomatik [17]. Eine Reihe weiterer Publikationen gibt für die CT bei der Identifizierung einer COVID-19-Erkrankung höhere Sensitivitäten an als für die RT-PCR. Dies gab Anlass zur Empfehlung einzelner Autoren und Autorinnen, die CT zur Diagnose von COVID19 einzusetzen, insbesondere, wenn eine RT-PCR aufgrund eines zu hohen Bedarfs nicht verfügbar oder infolge einer Überlastung der Logistik und der Labore die Wartezeit auf ein Ergebnis unvertretbar lang ist $[18,19]$.

Im Zusammenhang mit der SARS-CoV2-Pandemie wird eine bildgebende Untersuchung mit Hilfe einer Thorax-Computertomographie unter den folgenden Szenarien diskutiert:

- bei medizinisch asymptomatischen Personen ohne bekannten Kontakt mit an COVID-19-Erkrankten zum Nachweis einer Infektion mit SARSCoV-2 (Früherkennung),

- bei medizinisch asymptomatischen Kontaktpersonen von COVID-19-Erkrankten zum Nachweis einer Infektion mit SARS-CoV-2,

- bei symptomatischen Patienten und Patientinnen zur Sicherung der Diagnose COVID-19,

- bei symptomatischen Patienten und Patientinnen zur Unterstützung klinischer Entscheidungen. Diese können die Frage der stationären Aufnahme, therapeutischer Maßnahmen oder einer eventuellen Entlassung aus dem Krankenhaus betreffen.

\subsubsection{Betrachtung der Evidenzlage} Grundsätzlich ist die CT geeignet, Zeichen einer Erkrankung an COVID-19 und möglicherweise auch einer zunächst asymptomatischen Infektion mit SARS-CoV-2 nachzuweisen. Eine Vielzahl von retrospektiven Studien widmet sich diesem Thema, insbesondere einem Vergleich der Leistungsfähigkeit mit der der RT-PCR. So zeigt eine Metaanalyse von 68 Studien aus verschiedenen Ländern eine gepoolte Sensitivität von $94 \%$ für die CT (aus einem Bereich von 47 bis $100 \%$ ) und $89 \%$ für die RTPCR (Bereich: 40 bis 100\%). Die gepoolte Spezifität der CT betrug 37\% (Bereich: 25 bis $56 \%$ ). Für die RT-PCR wurde als Goldstandard zwar grundsätzlich eine Spezifität von $100 \%$ angenommen, aber für die Abschätzung von Prädiktionswerten wurde ihr ein Wert von 99\% zugewiesen. Die Prävalenzder Erkrankung streute zwischen 1 und 22,9\%. Die limitierte Spezifität der CT in Verbindung mit der schwankenden Prävalenz unter den untersuchten Personen erklärt, dass der positive Vorhersagewert (PPV) über einen breiten Bereich streute, für die CT zwischen 1,5 und 30,7\%. Für die RT-PCR liegt er unter Annahme einer Spezifität von $99 \%$ zwischen 43,7 und 96,4\%.Dernegative Vorhersagewert (NPV) betrug sowohl für die CT als auch die RTPCR mehr als 95\% [20]. Übereinstimmende Befunde finden sich bei $\mathrm{Xu}$ et al. [21]. Die Metaanalyse von Kim et al. [20] ergab für die eingeschlossenen deutschen Studien an selektierten Populationen (Stand 29. März 2020) eine mittlere Prävalenz von $5,7 \%$. Hieraus ergaben sich geschätzt für die CT ein PPV von $8,3 \%$ und ein NPV von $99 \%$, und für die RT-PCR ein PPV von $84,3 \%$ und ein NPV von 99,3\%.

Dabei handelt es sich durchweg um retrospektive Analysen, deren Interpretation insofern schwierig ist, da nicht erkennbar ist, bei welchen Erkrankten und mit welchem Zweck die CT erfolgte: Erkrankungsnachweis bei unklarer Symptomatik im Frühstadium oder wegen Verdachts auf manifeste COVID-19-Pneumonie bei schwerer Symptomatik mit oder ohne vorliegendem SARS-CoV-2-Nachweis. Ebenso wenig sind die Prävalenz von COVID-19 im untersuchten Kollektiv und die jeweilige Prät-Test-Wahrscheinlichkeit bekannt. Insbesondere die Prävalenz hat erheblichen Einfluss auf den positiven Vorhersagewert der Untersuchung.

Die Spezifität der CT ist gering, sodass COVID-19-bedingte pulmonale Infiltrate nicht ausreichend von interstitiellen Pneumonien anderer Ursache unterschieden werden können. Gerade in einer Grippesaison stellt dies ein erhebliches Problem dar. Außerdem berichten spätere Studien über unauffällige CT-Befunde bei ca. $50 \%$ der Erkrankten, insbesondere in den frühen Krankheitsphasen, so dass die in vielen Studien berichtete hohe Sensitivität der CT nicht unwidersprochen ist. Worin die Ursache für die Diskrepanzen besteht, ist unklar. Möglicherweise waren die Indikation zur Durchführung der CT, die Definition eines Krankheitsverdachts oder des Schweregrads der Symptomatik nicht einheitlich.

Eine Readerstudie (medizinisch-diagnostische Studie mit einer begrenzten, festen Anzahl von Befundern) mit drei Radiologen oder Radiologinnen ergab zwar, dass diese mit einer Treffsicherheit zwischen 60 und $83 \%$ zwischen einer COVID-19- und einer Nicht-COVID19-Pneumonie unterscheiden konnten [22], aber ob sich die artifiziellen Bedingungen einer Readerstudie auf die Praxis übertragen lassen, bleibt unklar. Dieselbe Forschergruppe berichtet auch, dass eine ergänzende Unterstützung der Diagnose mittels eines Deep-Learning-Algorithmus zusätzlich zur Befundung durch Radiologen oder Radiologinnen die Treffsicherheit verbessern konnte [23]. Somit können auch Verfahren der künstlichen Intelligenz (KI) zu einer Unterstützung und Verbesserung der Diagnostik beitragen, aber die Auswirkungen ihres Einsatzes auf das Problem der eingeschränkten Spezifität der Lungen-CT sind gering.

\subsubsection{Abwägung der Indikationen zur CT der Lunge}

Bei Patienten und Patientinnen mit dringendem Verdacht auf eine COVID-19Pneumonie unterliegt wie bei anderen Pneumonien die Durchführung einer CT der rechtfertigenden Indikation durch einen Arzt oder eine Ärztin mit der erforderlichen Fachkunde im Strahlenschutz. 
Tab. 1 Gegenüberstellung der Empfehlungen der WHO [24] und der Stellungnahme der SSKzur CT-Diagnostik bei einer COVID-19-Erkrankung

\begin{tabular}{|c|c|c|c|c|}
\hline & \multicolumn{3}{|l|}{ WHO } & \multirow{2}{*}{$\begin{array}{l}\text { SSK } \\
\text { Stellungnahme der SSK für } \\
\text { Deutschland }\end{array}$} \\
\hline & Personenkreis & $\begin{array}{l}\text { Zweck der bildge- } \\
\text { benden Diagnostik }\end{array}$ & Empfehlung der WHO & \\
\hline R1 & $\begin{array}{l}\text { Kontaktpersonen von } \\
\text { COVID-19-Erkrankten ohne } \\
\text { Symptome }\end{array}$ & $\begin{array}{l}\text { Nachweis der SARS- } \\
\text { CoV-2-Infektion }\end{array}$ & Nicht empfohlen & $\begin{array}{l}\text { Aus fachlicher Sicht der SSK } \\
\text { nicht empfohlen und wegen } \\
\text { fehlender rechtfertigender } \\
\text { Indikation nicht zulässig }\end{array}$ \\
\hline R2.1 & $\begin{array}{l}\text { Symptomatisch Erkrankte } \\
\text { mit Verdacht auf COVID-19 }\end{array}$ & $\begin{array}{l}\text { Nachweis von COVID- } \\
19\end{array}$ & $\begin{array}{l}\text { Nicht empfohlen, wenn Ergebnis der RT-PCR in vertret- } \\
\text { barer Zeit verfügbar ist }\end{array}$ & $\begin{array}{l}\text { Aus fachlicher Sicht der SSK } \\
\text { nicht empfohlen und wegen } \\
\text { fehlender rechtfertigender } \\
\text { Indikation nicht zulässig }\end{array}$ \\
\hline $\mathrm{R} 2.2$ & $\begin{array}{l}\text { Symptomatisch Erkrankte } \\
\text { mit Verdacht auf COVID-19 }\end{array}$ & $\begin{array}{l}\text { Nachweis von COVID- } \\
19\end{array}$ & $\begin{array}{l}\text { Empfohlen, wenn (1) RT-PCR nicht verfügbar ist, (2) ihr } \\
\text { Ergebnis erst nach unvertretbar langer Zeit verfügbar } \\
\text { ist, oder (3) die RT-PCR Ergebnisse initial negativ waren, } \\
\text { aber ein starker klinischer Verdacht fortbesteht }\end{array}$ & $\begin{array}{l}\text { Nur nach individueller } \\
\text { rechtfertigender Indikati- } \\
\text { on }\end{array}$ \\
\hline R3 & $\begin{array}{l}\text { Ambulante Patienten/ } \\
\text { Patientinnen mit vermute- } \\
\text { ter oder gesicherter COVID- } \\
19 \text { und leichten Sympto- } \\
\text { men }\end{array}$ & $\begin{array}{l}\text { Entscheidung über } \\
\text { stationäre Aufnahme }\end{array}$ & $\begin{array}{l}\text { Empfohlen zusätzlich zur klinischen und laborchemi- } \\
\text { schen Diagnostik }\end{array}$ & $\begin{array}{l}\text { Nur nach individueller } \\
\text { rechtfertigender Indikati- } \\
\text { on }\end{array}$ \\
\hline R4 & $\begin{array}{l}\text { Ambulante Patienten/ } \\
\text { Patientinnen mit vermute- } \\
\text { ter oder gesicherter COVID- } \\
19 \text { und mäßigen bis schwe- } \\
\text { ren Symptomen }\end{array}$ & $\begin{array}{l}\text { Entscheidung über } \\
\text { stationäre Aufnah- } \\
\text { me auf entweder } \\
\text { Normal- oder Inten- } \\
\text { sivstation }\end{array}$ & $\begin{array}{l}\text { Empfohlen zusätzlich zur klinischen und laborchemi- } \\
\text { schen Diagnostik }\end{array}$ & $\begin{array}{l}\text { Nur nach individueller } \\
\text { rechtfertigender Indikati- } \\
\text { on }\end{array}$ \\
\hline R5 & $\begin{array}{l}\text { Stationäre Patienten/ } \\
\text { Patientinnen mit vermute- } \\
\text { ter oder gesicherter COVID- } \\
19 \text { und mäßigen bis schwe- } \\
\text { ren Symptomen }\end{array}$ & $\begin{array}{l}\text { Unterstützung von } \\
\text { Entscheidungen zum } \\
\text { therapeutischen } \\
\text { Vorgehen }\end{array}$ & $\begin{array}{l}\text { Empfohlen zusätzlich zur klinischen und laborchemi- } \\
\text { schen Diagnostik }\end{array}$ & $\begin{array}{l}\text { Nur nach individueller } \\
\text { rechtfertigender Indikati- } \\
\text { on }\end{array}$ \\
\hline R6 & $\begin{array}{l}\text { Stationäre Patienten/ } \\
\text { Patientinnen mit COVID- } \\
\text { 19, deren Symptome in } \\
\text { Rückbildung sind }\end{array}$ & $\begin{array}{l}\text { Entscheidung über } \\
\text { die Entlassung }\end{array}$ & Nicht empfohlen & $\begin{array}{l}\text { Nur nach individueller } \\
\text { rechtfertigender Indikati- } \\
\text { on }\end{array}$ \\
\hline
\end{tabular}

Voraussetzung ist, dass das Ergebnis der CT klinische Entscheidungen beeinflusst und damit der Nutzen das Risiko überwiegt. Ein Einsatz der CT bei medizinisch asymptomatischen Personen, auch wenn sie einen Kontakt mit einem Infizierten hatten, ist nach deutschem Strahlenschutzrecht nicht zulässig und wird von der SSK auch als nicht gerechtfertigt angesehen: weder zur Abklärung des Verdachts auf COVID19 noch in Ergänzung zur oder anstelle der RT-PCR.

Die WHO publizierte am 11. Juni 2020 Empfehlungen zur Thoraxbildgebung bei COVID-19 [24], die vor dem Hintergrund der epidemiologischen Lage im ersten Halbjahr 2020 erarbeitet wurden. Zu diesem Zeitpunkt war das Wissen über die Übertragungswege und Dynamik der Pandemie noch begrenzt. Zudem stellte gerade während ihrer Anfangsphase die Knappheit von sowohl persönlicher Schutzausrüstung als auch Testkapazitä- ten ein ernstes Problem dar, das international verschieden ausgeprägt war. Seither ist vieles hinsichtlich der Übertragung klarer geworden (z.B. Überdispersion ${ }^{3}$, Superspreading-Ereignisse, Übertragung über Aerosole), und die Versorgung mit Atemschutz sowie die verfügbaren Testkapazitäten haben sich zumindest in Deutschland größtenteils stabilisiert. In - Tab. 1 wird abgewogen, inwieweit die wichtigsten Empfehlungen der WHO nach aktuellem Kenntnisstand und unter den in Deutschland herrschenden Bedingungen anwendbar sind.

Die WHO stellt ihren Empfehlungen zusätzliche Erläuterungen an die Seite, in de-

\footnotetext{
3 Überdispersion: Überdispersion beschreibt das Phänomen einer hohen Individuen-spezifischen Variation in der Verteilung der Anzahl der Sekundärübertragungen, die zu "Superverbreitungsereignissen“ (Superspreading) führen kann.
}

nen v. a. der Personenkreis präzisiert wird, der von der bildgebenden Diagnostik profitieren könnte. Nach Auffassung der SSK können die WHO-Empfehlungen zwar als Hinweise dahingehend verstanden werden, bei welchen Patienten und Patientinnen unter welchen Fragestellungen eine bildgebende Diagnostik grundsätzlich sinnvoll sein kann. Sie sind jedoch nur als "Vorschläge" zu verstehen. In jedem Fall bedarf es der individuellen, rechtfertigenden Indikation durch den fachkundigen Arzt oder die fachkundige Ärztin, einer Abwägung des Nutzens und des Risikos sowie einer Prüfung, ob die erwartete Information aus der Untersuchung für eine anstehende Entscheidung notwendig ist, und ob diese Information auch ohne Anwendung ionisierender Strahlung gewonnen werden kann.

Ein Nachweis COVID-19-typischer pulmonaler Veränderungen bei einer Symptomatik allein der oberen Atemwege bedingt 
nach den derzeit geltenden Empfehlungen keine anderen Maßnahmen als diejenigen, die ohne CT-Befund empfohlen würden: häusliche Isolation und symptomatische Behandlung und Wiederholung der RT-PCR, falls der erste Test negativ war. Mit der Verfügbarkeit von Antigen-Schnelltests dürften solche Entscheidungen für oder wider therapeutische oder seuchenhygienische Maßnahmen noch einfacher werden. Auch sind derzeit keine Interventionen bekannt, durch die sich im weiteren Verlauf durch einen CT-Befund eine klinisch manifeste COVID-19-Pneumonie und schlimmstenfalls ein schwerer Krankheitsverlauf abwenden oder abmildern ließen.

Die Fleishner-Society ${ }^{4}$ gibt Empfehlungen zum Einsatz der $\mathrm{CT}$ für drei Szenarien, unter Berücksichtigung der Verfügbarkeit von RT-PCR-Tests, von Antigen-Schnelltests sowie der Prä-TestWahrscheinlichkeit einer Infektion. Diese Wahrscheinlichkeit ist bei sporadischem Kontakt zu einer infizierten Person als niedrig einzustufen, bei einer Exposition in einer Clustersituation als intermediär und bei einer erkrankten Person im Haushalt als hoch. Neben der WHO sieht auch die Fleishner-Society unabhängig von Risikofaktoren und Testergebnissen eine klare Indikation zur CT bei mäßiger oder schwerer Symptomatik sowie einer signifikanten Verschlechterung anfangs leichter Symptome zur Erhebung eines Ausgangsbefunds für den weiteren Verlauf und zum Nachweis möglicher anderer Ursachen. $\mathrm{Zu}$ Indikationen bei leichter Symptomatik, z.B. beim Vorhandensein bestimmter Risikofaktoren oder einer hohen Prä-TestWahrscheinlichkeit, gibt sie keine definitive Empfehlung; Entscheidungen sind nur im Einzelfall möglich.

Auch bei Erkrankten mit persistierenden Symptomen nach überstandener Erkrankung, insbesondere nach vorübergehender Intensiv- oder Beatmungspflicht, kann eine $\mathrm{CT}$ indiziert sein, um Kompli-

\footnotetext{
${ }^{4}$ Die Fleishner Society ist eine internationale, multidisziplinäre Fachgesellschaft, die die Erforschung und Verbesserung der Thoraxradiologie zum Ziel hat. Ihre Empfehlungen sind international hoch angesehen und finden bei diversen Leitlinien zur Thoraxbildgebung Berücksichtigung.
}

kationen nachzuweisen, die medizinische Maßnahmen erfordern.

Übereinstimmend raten sowohl einzelne Wissenschaftler und Wissenschaftlerinnen als auch Fachgesellschaften (American College of Radiology, ACR; The Royal College of Radiologists, RCR; deutsche Röntgengesellschaft, DRG) und nationale und internationale Institutionen (WHO; Centers for Disease Control and Prevention, $\mathrm{CDC}$ ) vom Einsatz der CT bei bloßem Krankheitsverdacht ab [24-31]. Für Kinder geben die entsprechenden Fachgesellschaften (Gesellschaft für Pädiatrische Radiologie, GPR; European Society of Pediatric Radiology, ESPR) eigenständige Empfehlungen $a b$ [32]. Der Einsatz röntgendiagnostischer Verfahren bei symptomatisch Erkrankten hingegen ist davon unberührt, sofern sie als Baseline vor einer befürchteten klinischen Verschlechterung oder als Grundlage für eine klinische Entscheidung benötigt werden, einschließlich der über eine stationäre Aufnahme oder eine ambulante Weiterbehandlung. Bei schwer Erkrankten gehören Röntgenaufnahmen und CT-Scans der Lunge zur diagnostischen Routine und werden angeordnet, um auf ein klinisches Problem angemessen reagieren zu können. Von regelmäßigen Röntgenthorax- oder CT-Kontrollen ohne konkreten Anlass wird indes auch beim Intensiv- oder Beatmungspatienten übereinstimmend abgeraten [31].

\subsubsection{Technische Empfehlungen zur Optimierung der CT-Diagnostik bei COVID-19}

Die CT sollte möglichst in "Low-dose"Technik durchgeführt werden. Unter "Lowdose"-Computertomographie (LDCT) wird hierbei eine diagnostische computertomographische Röntgenuntersuchung des Thorax verstanden, deren Einstellparameter gemäß Larke et al. [33] zu einer durchschnittlichen Organdosis der Lunge von maximal $4,5 \mathrm{mSv}$ und einer durchschnittlichen effektiven Dosis von maximal 1,4 mSv führen. Weiterhin müssen hier geschlechtsspezifische Werte berücksichtigt werden. Im Zuge laufender und künftiger Weiterentwicklungen der CT-Technik können sich diese Anhaltszahlen durchaus noch ändern. Da die intrinsischen Kontraste der Lunge hoch sind, ist eine LDCT bei normalgewichtigen Personen möglich, ohne dass diagnostisch wichtige Information verloren geht. Anzustreben ist bei schlanken und normal gebauten Personen ein Volumen-CT-Dosisindikator (CTDlvol) von deutlich unter $3 \mathrm{mGy}$. Kang et al. konnten zeigen, dass mit modernen Geräten und einem für niedrige Dosis optimierten Protokoll entsprechende Aufnahmen mit einem CTDlvol von nur $0,4 \mathrm{mGy}$ möglich sind, gegenüber einem CTDIvol von ca. $3,4 \mathrm{mGy}$ mit demselben Gerät bei Verwendung des Standardprotokolls [34]. Bei adipösen oder sehr muskulösen Personen können höhere oder Standarddosen unvermeidlich sein [35]. Um eine Hochregelung der Dosis zu vermeiden, sollte versucht werden, bei den zu untersuchenden Personen die Arme so weit wie möglich über den Kopf zu lagern, damit die Schultergelenke kranial der Lungenspitzen gelegen sind und nicht vom Scan erfasst werden. Der Scan ist bei voller Einatmung streng auf den Bereich zwischen Lungenspitzen und Recessus costodiaphragmaticus zu begrenzen und nicht auf die Oberbauchorgane auszudehnen, wie beim RoutineCT des Thorax üblich. Zusätzliche Aufnahmen in Exspiration sind nicht indiziert. Neben dem Topogramm ist nur eine einzelne, native Spirale erforderlich. Die Möglichkeiten, die der Spiralscanner zur Dosissenkung bietet (Pitch, Aufnahmespannung, Röhrenstrom), sollten sinnvoll genutzt werden. Empfohlen wird eine Schichtdicke von $1 \mathrm{~mm}$ mit überlappender Rekonstruktion. Bei Erkrankten mit Atemnot, die nicht über längere Zeit die Luft anhalten können, sind bei älteren Geräten ggf. eine größere Schichtdicke und/oder ein größerer Pitch erforderlich, um die Scanzeit zu begrenzen.

Bei Verdacht auf Thromboembolie der Lungengefäße ist ein kontrastmittelverstärktes, einphasiges Lungenembolie-Protokoll zu verwenden, wie es in den meisten radiologischen Einrichtungen etabliert ist. Eine zusätzliche Nativserie oder Aufnahme in Dual-Energy-Technik mit zwei Röntgenröhren ist nicht erforderlich. Da bei dieser Indikation die Dichteauflösung im Vordergrund steht, sind eine höhere Schichtdicke (typischerweise $3 \mathrm{~mm}$ ) und eine Rekonstruktion mit einem Weichteilalgorithmus zusätzlich zum kantenbetonten Algorithmus erforderlich, der für das Lungenpa- 
renchym angewendet wird. Bei Mehrzeilengeräten können aus dem Rohdatensatz eines Scans sowohl 1-mm- als auch 3-mmSchichten rekonstruiert werden. Falls diese Möglichkeit nicht besteht, sollte die Beurteilung des Lungenparenchyms anhand der 3-mm-Schichten erfolgen und kein zusätzlicher 1-mm-Scan angefertigt werden.

\subsubsection{Nutzen-Risiko-Abwägung zur CT der Lunge bei COVID-19}

Die Risiken der CT sind für die einzelne Person vergleichsweise gering. Mit dem Ausbreitungsgrad der COVID-19-Pandemie ist jedoch die resultierende Kollektivdosis relevant für den Strahlenschutz.

Ferner entstehen nicht strahlenbedingte Risiken für das medizinische Personal und andere Personen in derselben Institution. Das Infektionsrisiko für das Personal ist offensichtlich und hängt von der Schutzausrüstung ab und davon, wie nah die Betreffenden am Patienten oder der Patientin arbeiten müssen. Das technische Personal, das z.B. die Patienten und Patientinnen lagert, kann einem Körperkontakt nicht ausweichen. Andere Patienten und Patientinnen sind aufgrund ihrer eigenen Erkrankung häufig Risikopersonen, für die eine Infektion besonders gefährlich ist. Indirekte Risiken entstehen, indem die Betriebsabläufe in der CT durch die Untersuchung (möglicherweise) infizierter Personen empfindlich gestört werden. So ist zwischen einzelnen Untersuchungen (möglicherweise) infizierter Personen eine gründliche Desinfektion und Lüftung erforderlich, was zwangsläufig den Abstand zwischen zwei Untersuchungen erhöht und zu Engpässen bei der Kapazität für CT-Untersuchungen aus anderer Indikation führen kann.

CT-Untersuchungen medizinisch asymptomatischer Personen, auch wenn sie Kontakt mit einer infizierten Person hatten, sind aus fachlicher Sicht derzeit nicht gerechtfertigt und nach deutschem Strahlenschutzrecht unzulässig. Auch bei einer CT-Untersuchung allein wegen eines COVID-19-Verdachts steht den genannten Risiken kein nennenswerter Individualoder Kollektivnutzen gegenüber, solange die Konsequenzen eines positiven CT-Befundes nicht über die ohnehin empfohlenen bzw. vom Gesundheitsamt angeordneten Maßnahmen hinausge- hen. In Einzelfällen kann dies zutreffen [36], woraus sich ggf. eine individuelle rechtfertigende Indikation ergibt.

An dieser Stelle sei auch kritisch auf die Möglichkeiten des Einsatzes von KI hingewiesen. In der diagnostischen Anwendung ionisierender Strahlung müssen prinzipiell zwei verschiedene KI-Anwendungen unterschieden werden. Zum einen ist es möglich, mit Hilfe von KI nichtlineare Rekonstruktionsverfahren zu optimieren und zu beschleunigen und damit diagnostisch aussagekräftige CT-Bilder mit niedrigeren Strahlendosen zu erhalten, als für die derzeit verwendeten Rekonstruktionsverfahren erforderlich ist. Dies setzt jedoch voraus, dass die Algorithmen anhand von typischen pathologischen Befunden in einer großen Zahl von Bilddatensätzen trainiert worden sind. Unbekannte Strukturen würden sonst z. B. als Rauschen interpretiert und unterdrückt, oder auch übertrieben oder gänzlich falsch dargestellt. Entsprechende Ansätze für spezialisierte Rekonstruktionsalgorithmen für die CT bei COVID-19-Erkrankten finden sich in der Literatur derzeit nicht. Unabhängig davon ist dieser Einsatz von KI insofern problematisch, als es nicht möglich ist, sich von der Echtheit der damit erzeugten Bilder zu überzeugen, da aufgrund der vergleichsweise niedrigen Strahlendosis und des damit sehr hohen Rauschanteils mit konventionellen Rekonstruktionsverfahren keine aussagekräftigen Referenzbilder berechnet werden können. Der zweite Anwendungsbereich von $\mathrm{KI}$ ist die Diagnoseunterstützung durch automatisierte Auswertung der bildgebenden Untersuchungen. Bereits in Abschn. 3.2.3 wurde ein entsprechender Ansatz aus der Literatur angeführt. Allerdings sind diesbezüglich die Fallzahlen noch zu klein, um einen derartigen Ansatz zuverlässig bewerten zu können. Grundsätzlich aber kann eine automatisierte Zweitbefundung mit Hilfe von Methoden der KI durchaus eine sinnvolle Unterstützung darstellen, insbesondere, wenn eine große Zahl von Untersuchungen zu bewältigen ist und wenn durch den Einsatz von KI eine geringere Anzahl erfahrener Radiologen und Radiologinnen mit der Befundung betraut werden müssen. Dennoch ist eine kritische Bewertung von automatisch erzeugten Diagnosen durch erfahrene Radiolo- gen und Radiologinnen unerlässlich. Eine ausschließlich automatisierte Befundung wird derzeit nicht empfohlen, zumal die Anzahl für das Training der Algorithmen zur Verfügung stehender Bilddatensätze niedrig und das Wissen über mögliche Besonderheiten der Merkmale der Atemwegserkrankungen bei COVID-19-Erkrankten noch begrenzt ist. Sinnvoll wäre der Einsatz von KI z. B. im Rahmen der Stratifizierung von COVID-19-Erkrankten zur Vorhersage der Schwere des Krankheitsverlaufs oder potenziell sinnvoller Behandlungsmethoden. Auch eine Kopplung z.B. mit Methoden der röntgenbasierten Dunkelfeldbildgebung [37] wäre denkbar. Entsprechende Studien mit einer ausreichenden Anzahl an Patienten und Patientinnen und angemessener Nachbeobachtung aufzusetzen oder gar abzuschließen, ist unter den gegenwärtigen Bedingungen der Pandemie allerdings unrealistisch.

\section{Rechtliche Rahmenbedingungen}

Gemäß § 83 Abs. 1 StrlSchG dürfen ionisierende Strahlung und radioaktive Stoffe am Menschen (im Folgenden kurzStrahlenanwendung am Menschen genannt) nur ...

1. im Rahmen einer medizinischen Exposition oder

2. zur Untersuchung einer Person in durch Gesetz vorgesehenen oder zugelassenen Fällen oder nach Vorschriften des allgemeinen Arbeitsschutzes oder nach Einwanderungsbestimmungen anderer Staaten (nichtmedizinische Anwendung) angewendet werden.

Der Begriff medizinische Exposition beinhaltet u.a. (vgl. § 2 Abs. 8 StrlSchG) eine Strahlenanwendung ...

- an einem Patienten oder einer Patientin zu dessen bzw. deren medizinischer oder zahnmedizinischer Untersuchung oder Behandlung,

- an einer asymptomatischen Person zur Früherkennung einer bestimmten Erkrankung,

- an einer Person zum Zweck der medizinischen Forschung.

Untersuchungen mit Röntgenstrahlung nach dem Infektionsschutzgesetz [38] unterfallen nicht dem Begriff der medizinischen Exposition, sondern dem der 
nichtmedizinischen Anwendung (§ 83 Abs. 1 Nr. 2 StrlSchG).

Eine Strahlenanwendung am Menschen muss einen hinreichenden Nutzen erbringen. Bei der Bewertung, ob die Anwendung einen hinreichenden Nutzen erbringt, ist ihr Gesamtpotenzial an diagnostischem oder therapeutischem Nutzen, einschließlich des unmittelbaren gesundheitlichen Nutzens für den Einzelnen und des Nutzens für die Gesellschaft, gegen die von der Exposition möglicherweise verursachte Schädigung des Einzelnen abzuwägen ( $\S 83$ Abs. 2 StrlSchG).

Grundsätzlich darf eine Strahlenanwendung am Menschen gemäß $\S 83$ Abs. 3 StrlSchG erst durchgeführt werden, nachdem ein Arzt oder eine Ärztin bzw. ein Zahnarzt oder eine Zahnärztin mit der erforderlichen Fachkunde im Strahlenschutz - auf Basis der oben beschriebenen Nutzen-Risiko-Abwägung entschieden hat, dass und auf welche Weise die Anwendung durchzuführen ist (sog. Stellung der rechtfertigenden Indikation). Darüber hinaus hat der/die die rechtfertigende Indikation stellende Arzt/ Ärztin oder Zahnarzt/Zahnärztin gemäß $\S 119$ Abs. 1 Strahlenschutzverordnung [39] zu prüfen, ob es sich bei der vorgesehenen Strahlenanwendung am Menschen um ein anerkanntes Verfahren nach den Erfordernissen der medizinischen Wissenschaften oder um einen Heilversuch handelt, dessen Durchführung durch den Arzt oder die Ärztin bzw. den Zahnarzt oder die Zahnärztin jedoch besonders zu begründen ist.

Die Pflicht zur Stellung der rechtfertigenden Indikation gemäß $\S 83$ Abs. 3 StrlSchG gilt nicht für Untersuchungen mit Röntgenstrahlung nach dem Infektionsschutzgesetz und für Anwendungen am Menschen zum Zweck der medizinischen Forschung nach $\S 31$ Abs. 1 oder $\S 32$ Abs. 1. Eine Anwendung im Rahmen der medizinischen Forschung bedarf jedoch einer Genehmigung durch das Bundesamt für Strahlenschutz (BfS) bzw. ist dem BfS vorher schriftlich oder elektronisch anzuzeigen.

Das Strahlenschutzgesetz definiert den Begriff Früherkennung als Anwendung von Röntgenstrahlung oder radioaktiven Stoffen im Rahmen einer medizinischen
Exposition zur Untersuchung von Personen, die keine Krankheitssymptome und keinen konkreten Krankheitsverdacht aufweisen (sog. asymptomatische Personen), um eine bestimmte Krankheit festzustellen ( $\$ 5$ Abs. 16 StrlSchG). Dabei wird unterschieden zwischen einer Früherkennung zur Ermittlung nichtübertragbarer Krankheiten (z.B. Früherkennung von Brustkrebs) und einer Früherkennung zur Ermittlung übertragbarer Krankheiten in Landesteilen oder für Bevölkerungsgruppen mit überdurchschnittlicher Erkrankungshäufigkeit. Letztere Form der Früherkennung ist gemäß $\S 84$ Abs. 4 StrISchG nur zulässig, wenn die zuständige oberste Landesgesundheitsbehörde im Einvernehmen mit der obersten Strahlenschutzbehörde des Landes eine Früherkennungsuntersuchung zur öffentlichen Gesundheitsvorsorge zugelassen hat.

Wie jede Strahlenanwendung am Menschen unterliegt auch eine Exposition zur Untersuchung oder Behandlung einer COVID-19-Erkrankung dem oben dargelegten rechtlichen Rahmen gemäß StrISchG und StrISchV.

\section{Korrespondenzadresse}

Strahlenschutzkommission (SSK)

Geschäftsstelle der Strahlenschutzkommission Robert-Schuman-Platz 3, 53175 Bonn, Deutschland

info-ssk@bfs.de

\section{Einhaltung ethischer Richtlinien}

Interessenkonflikt. Die Strahlenschutzkommission erklärt, dass die Autoren keine Interessenkonflikte haben.

Für diesen Beitrag wurden von den Autoren keine Studien an Menschen oder Tieren durchgeführt. Für die aufgeführten Studien gelten die jeweils dort angegebenen ethischen Richtlinien.

\section{Literatur}

1. StrlSchG (2017) Gesetz zum Schutz vor der schädlichen Wirkung ionisierender Strahlung (Strahlenschutzgesetz - StrlSchG) vom 27. Juni 2017 (BGBI. I S. 1966), das zuletzt durch Artikel 11 des Gesetzes vom 12. Dezember 2019 (BGBI. I S. 2510) geändert worden ist

2. Plante JA, Liu Y, Liu J et al (2021) Spike mutation D614G alters SARS-CoV-2 fitness. Nature 592(7852):116-121

3. World Health Organization (WHO) (2003) Cumulative Number of Reported Probable Cases of SARS https://www.who.int/csr/sars/country/2003_07_ 11/en/.Zugegriffen: 10.02.2021

4. Chafekar A, Fielding BC (2018) MERS-CoV: Understanding the Latest Human Coronavirus Threat. Viruses 10(2):24

5. Fan Y, Zhao K, Shi ZL et al (2019) Bat Coronaviruses in China. Viruses 11(3):E210. https://doi.org/10. 3390/v11030210

6. Dodt C, Schneider N (2020) Emergency and intensive care medicine aspects of COVID-19 infections. Radiologe 60(10):899-907

7. Hemmer CJ, Geerdes-Fenge HF, Reisinger EC (2020) COVID-19: epidemiology and clinical facts. Radiologe 60(10):893-898

8. Kniep I, Lutter M, Ron A et al (2020) Postmortem imaging of the lung in cases of COVID-19 deaths. Radiologe 60(10):927-933

9. WHO (2020) WHO Coronavirus Disease (COVID-19) Dashboard. https://covid19.who.int. Zugegriffen: 10.02.2021

10. Robert Koch-Institut (RKI) (2020) COVID19: Fallzahlen in Deutschland und weltweit. https://www.rki.de/DE/Content/InfAZ/N/ Neuartiges_Coronavirus/Fallzahlen.html. Zugegriffen: 10.02.2021

11. Sheridan C (2020) Fast, portable tests come online to curb coronavirus pandemic. Nat Biotechnol 38(5):515-518

12. Denkinger $C$, Brümmer $L$, Katzenschlager $S$ (2020) Rapid antigen tests for the diagnosis of a SARSCoV-2 infection. https://diagnosticsglobalhealth. org. Zugegriffen: 10.02.2021

13. Heidinger $\mathrm{BH}$, Kifjak D, Prayer $\mathrm{F}$ et al (2020) Radiologicalmanifestations of pulmonarydiseases in COVID-19. Radiologe 60(10):908-915

14. Li K, Wu J, Wu F et al (2020) The Clinical and Chest CT Features Associated With Severe and Critical COVID-19Pneumonia. Invest Radiol55(6):327-331

15. Zhao W, Zhong Z, Xie X et al (2020) Relation Between Chest CT Findings and Clinical Conditions of Coronavirus Disease (COVID-19) Pneumonia: A Multicenter Study. AJR Am J Roentgenol 214(5):1072-1077

16. Prokop M, van Everdingen W, van Rees Vellinga T et al (2020) CO-RADS: A Categorical CT Assessment Scheme for Patients Suspected of Having COVID-19-Definition and Evaluation. Radiology 296(2):E97-E104

17. Guan WJ, Ni ZY, Hu Y et al (2020) Clinical Characteristics of Coronavirus Disease 2019 in China. NEngl J Med 382(18):1708-1720

18. Ai T, Yang Z, Hou Het al (2020) Correlation of Chest CT and RT-PCR Testing for Coronavirus Disease 2019 (COVID-19) in China: A Report of 1014 Cases. Radiology 296(2):E32-E40

19. Fang $Y$, Zhang $H$, Xie J et al (2020) Sensitivity of Chest CT for COVID-19: Comparison to RT-PCR. Radiology 296(2):E115-E117

20. Kim H, Hong H, Yoon SH (2020) Diagnostic Performance of CT and Reverse Transcriptase Polymerase Chain Reaction for Coronavirus Disease 2019: A Meta-Analysis. Radiology 296(3):E145-E155

21. Xu B, Xing Y, Peng J et al (2020) Chest CT for detecting COVID-19: a systematic review and meta-analysis of diagnostic accuracy. Eur Radiol 30(10):5720-5727

22. Bai HX, Hsieh B, Xiong Zetal (2020) Performance of Radiologists in Differentiating COVID-19from NonCOVID-19 Viral Pneumonia at Chest CT. Radiology 296(2):E46-E54

23. Bai HX, Wang R, Xiong $Z$ et al (2020) Artificial Intelligence Augmentation of Radiologist Performance in Distinguishing COVID-19 from 
Pneumonia of Other Origin at Chest CT. Radiology 296(3):E156-E165

24. World Health Organisation (WHO) (2020) Use of chest imaging in COVID-19: A rapid advice guide. https://www.who.int/publications/i/item/ use-of-chest-imaging-in-covid-19. Zugegriffen: 10.02.2021

25. American College of Radiology (ACR) (2020) Recommendations for the use of Chest Radiography and Computed Tomography (CT) for Suspected COVID-19 Infection. https:// www.acr.org/Advocacy-and-Economics/ACRPosition-Statements/Recommendations-forChest-Radiography-and-CT-for-SuspectedCOVID19-Infection.Zugegriffen: 10.02.2021

26. Centers for Disease Control and Prevention (CDC) (2020) Interim Clinical Guidance for Management of Patients with Confirmed Coronavirus Disease (COVID-19). https://www.cdc.gov/coronavirus/ 2019-ncov/hcp/clinical-guidance-managementpatients.html.Zugegriffen: 10.02.2021

27. Cleverley J, Piper J, Jones MM (2020) The role of chest radiography in confirming covid- 19 pneumonia. BMJ 370:m2426

28. Li K, Fang Y, Li W et al (2020) CT image visual quantitative evaluation and clinical classification of coronavirus disease (COVID-19). Eur Radiol 30(8):4407-4416

29. The Royal College of Radiologists (RCR) (2020) Coronavirus (COVID-19) clinical radiology resources. https://www.rcr.ac.uk/college/ coronavirus-covid-19-what-rcr-doing/clinicalinformation/role-ct-chest/role-ct-patients. Zugegriffen: 10.02.2021

30. Revel MP, Parkar AP, Prosch H et al (2020) COVID-19 patients and the radiology department-advice from the European Society of Radiology (ESR) and the European Society of Thoracic Imaging (ESTI). Eur Radiol 30(9):4903-4909

31. Vogel-Claussen J, Ley-Zaporozhan J, Agarwal P et al (2020) Recommendations of the Thoracic Imaging Section of the German Radiological Society for clinical application of chest imaging and structured CT reporting in the COVID-19 pandemic. Rofo 192(7):633-640

32. Raissaki M, Shelmerdine SC, Damasio MB et al (2020) Management strategies for children with COVID-19: ESPR practical recommendations. Pediatr Radiol 50(9):1313-1323

33. Larke FJ, Kruger RL, Cagnon CH et al (2011) Estimated radiation dose associated with lowdose chest CT of average-size participants in the National Lung Screening Trial. AJR Am J Roentgenol 197(5):1165-1169

34. Kang Z, Li X, Zhou S (2020) Recommendation of low-dose $C T$ in the detection and management of COVID-2019. Eur Radiol 30(8):4356-4357

35. Kalra MK, Homayounieh F, Arru C et al (2020) Chest CT practice and protocols for COVID-19 from radiation dose management perspective. Eur Radiol 30(12):6554-6560

36. Rubin GD, Ryerson CJ, Haramati LB et al (2020) The Role of Chest Imaging in Patient Management during the COVID-19 Pandemic: A Multinational Consensus Statement from the Fleischner Society. Radiology 296(1):172-180

37. Willer K, Fingerle AA, Gromann LB et al (2018) $X$-ray dark-field imaging of the human lung-A feasibility study on a deceased body. PLOS ONE 13(9):e204565

38. IfSG (2000) Infektionsschutzgesetz vom 20. Juli 2000 (BGBI. I S. 1045), das zuletzt durch Artikel 4a des Gesetzes vom 21. Dezember 2020 (BGBI. I S.3136) geändert worden ist

\section{Medical applications of ionising radiation for the diagnosis of coronavirus-associated lung disease. Statement by the German Commission on Radiological Protection}

Issue: Since the start of the global pandemic, a number of publications have suggested using ionising radiation to diagnose a COVID-19 infection. Some proposals call for the use of computed tomography (CT) to screen for presymptomatic SARS-CoV-2infections.

Intent of the consultation: With these proposals in mind, the German Commission on Radiological Protection (SSK) agreed during its 307th meeting on 2 and 3 July 2020 to establish a working group aimed at evaluating the use of ionising radiation in connection with a COVID-19 infection. The working group focussed on radiological protection aspects with a view to using computed tomography to diagnose COVID-19. The group started its work on 5 October 2020. Although not commissioned by the Federal Ministry for the Environment, Nature Conservation and Nuclear Safety (BMU), representatives of the BMU and the Federal Office for Radiation Protection (BfS) were involved in the consultations.

Statement: The SSK issues the following statement: 1. The use of CT in asymptomatic persons to diagnose COVID-19 outside of approved studies is not evidentially justified. 2. Pursuant to Section 83 (3) of the German Radiation Protection Act, a CT scan for the diagnosis or follow-up of a COVID-19 pneumonia requires the prior justifying indication from a doctor with the requisite specialist knowledge in radiation protection. To this end, specialist medical associations provide recommendations based on up-to-date findings.

\section{Keywords}

Coronavirus · COVID-19 · SARS-CoV-2 · Computed Tomography $\cdot$ Radiation Protection

39. StrlSchV (2018) Verordnung zum Schutz vor der schädlichen Wirkung ionisierender Strahlung (Strahlenschutzverordnung - StrlSchV) vom 29. November 2018. BGBI. I S. 2034, 2036, die durch Artikel 1 der Verordnungvom 27. März 2020 (BGBI.I S.748) geändertworden ist 